\title{
GFAP, Ki67 and IDH1: perhaps the golden triad of glioma immunohistochemistry
}

\author{
Werner Paulus
}

Published online: 22 October 2009

(C) Springer-Verlag 2009

In this issue of Acta Neuropathologica, Capper et al. [2] describe a monoclonal antibody that specifically and sensitively recognizes the IDH1 (isocitrate dehydrogenase 1) protein carrying the $\mathrm{R} 132 \mathrm{H}$ mutation in routinely processed (formalin-fixed, paraffin-embedded) tissues. In 2008, hotspot sequence mutations at position 132 of $I D H I$ were discovered in $12 \%$ of glioblastomas [4] and in about $70 \%$ of diffuse astrocytomas, oligodendrogliomas, oligoastrocytomas and secondary glioblastomas [1], a finding that has been confirmed by several other studies. Because more than $90 \%$ of $\mathrm{IDH} 1$ mutations are of the R132H type and mutations are assumed to be present in all tumor cells, this antibody is able to identify virtually all glioma cells in paraffin sections from the majority of grade-II diffuse gliomas and from their more malignant descendants. When compared with a PCR- and restriction endonuclease-based assay for different $I D H I$ mutations recently described by the same group of authors [3], the immunohistochemical technique is easier to perform and is presumably more sensitive, whereas R132H-immunonegative cases will require additional PCR or sequencing analysis to search for other types of $I D H I$ and $I D H 2$ mutations. Although the antibody still needs to be evaluated by other institutions on a large number of specimens, it may represent one of the seminal achievements in diagnostic neuropathology of the past decades.

Hundreds of antibodies and antigens have been proposed as potential diagnostic markers for gliomas, but only a handful have turned out to be reliable and useful, the most important being GFAP for glial differentiation,

W. Paulus $(\bowtie)$

Institute of Neuropathology, University Hospital Münster,

Domagkstr. 19, 48129 Münster, Germany

e-mail: werner.paulus@uni-muenster.de developed in the seventies and Ki67/MIB1 for assessing proliferation, established in the eighties for frozen sections and in the nineties for paraffin sections. These antibodies, however, are not able to assist in resolving two common differential diagnostic problems: diffuse versus pilocytic astrocytoma; and invasion of diffuse astrocytoma versus reactive gliosis. Although staining for p53 is often performed to detect single-invading astrocytoma cells, it helps in only a minority of cases, in part because reactive astrocytes and other non-neoplastic cells may also show immunoreactivity for $\mathrm{p} 53$. In contrast, staining for R132H is expected to be of help in a significant fraction of cases and the diagnostic value of anti-R132H may reach that of anti-GFAP and MIB1.

The present work of Capper et al. is important for other reasons as well. First, because brain biopsies may be (rarely) associated with complications such as hemorrhage and infection, and because an ambiguous neuropathological diagnosis (invasive edge or gliosis) may lead to rebiopsy, increased diagnostic accuracy by demonstration of R132H-positive cells may decrease mortality and morbidity. Second, since IDHI mutations are associated with more favorable prognosis in diffuse astrocytic and oligodendroglial tumors of grades II-IV, staining for R132H (and for other IDHI mutations using antibodies to be developed) may provide important prognostic information. Third, the antibody will promote the detection of glioma cells in CSF specimens. Fourth, the availability of the mutation-specific antibody will stimulate research using biopsy materials by clearly identifying tumor cells and correlating them with a variety of structural and molecular features. Fifth, anti-R132H will be useful for biological experiments aimed at further elucidating the function of mutated IDH1 protein; and finally, this report illustrates the efficient and beneficial outcome of a close cooperation and 
institutional association between a neuropathology department and the infrastructure of a large scale biomedical research center, i.e. the German Cancer Research Center in Heidelberg.

In my view, anti-R132H antibodies should improve patient care, stimulate research and be indispensable for any institution involved in glioma research and diagnosis.

\section{References}

1. Balss J, Meyer J, Mueller W, Korshunov A, Hartmann C, von Deimling A (2008) Analysis of the IDH1 codon 132 mutation in brain tumors. Acta Neuropathol 116:597-602
2. Capper D, Zentgraf H, Balss J, Hartmann C, von Deimling A (2009) Monoclonal antibody specific for IDH1 R132H mutation. Acta Neuropathol (in press) PMID:19798509

3. Meyer J, Pusch S, Balss J, Capper D, Mueller W, Christians A, Hartmann C, von Deimling A (2009) PCR- and restriction endonuclease-based detection of IDH1 mutations. Brain Pathol (in press) PMID: 19744125

4. Parsons DW, Jones S, Zhang X et al (2008) An integrated genomic analysis of human glioblastoma multiforme. Science 321:18071812 